\title{
Inventario florístico de la Reserva Hídrica Natural "Río Carcarañà" en la comuna de Pueblo Andino (Santa Fe, Argentina)
}

\author{
Floristic inVentory of the Natural Water Reserve "Río \\ Carcarañá” in the municipality of Pueblo Andino (Santa Fe, \\ Argentina)
}

\author{
Eugenia Pedrero ${ }^{1,2 *}\left(\mathbb{C}\right.$, Mauro M. Torales ${ }^{1}\left[\right.$ y Germán Saigo ${ }^{1}[$
}

1. Museo Provincial de Ciencias Naturales "Dr. Ángel Gallardo", San Lorenzo 1949, Rosario, Argentina.

2. Facultad de Ciencias Agrarias, Universidad Nacional de Rosario (UNR), Campo Experimental Villarino, C.C. 14 (S2125ZAA) Zavalla, Argentina.

*peugenia@hotmail.com.ar

\section{Citar este artículo}

PEDRERO, E., M. M. TORALES \& G. SAIGO. 2022. Inventario florístico de la Reserva Hídrica Natural "Río Carcarañá" en la comuna de Pueblo Andino (Santa Fe, Argentina). Bol. Soc. Argent. Bot. 57: 117-130.

DOI: https://doi. org/10.31055/1851.2372.v57. n1.33926

Recibido: 20 Jul 2021

Aceptado: 17 Dic 2021

Publicado en línea: 20 Feb 2022

Publicado impreso: 31 Mar 2022

Editor: Diego Gutiérrez

ISSN versión impresa 0373-580X

ISSN versión on-line 1851-2372

\section{SUMmaRY}

Background and aims: The Natural Water Reserve "Río Carcarañá", included within the Pampean Phytogeographic Province, is one of the few protected areas in the southern region of Santa Fe province, one which has been profoundly modified by agricultural and cattle activities. Floristic studies within the reserve have been conducted mostly over the area named "El Espinillo", describing it as a relictual formation of native vegetation related to the Espinal Phytogeographic Province. The objectives of this article are to make a floristic survey within the boundaries of Pueblo Andino town (Iriondo department), to describe the family diversity and, to identify native and exotic species as well as relevant ones due to the phytogeographical context of the study area.

M\&M: Field work took place during nine visits made to the area between 2014 and 2021 , where specimens were collected in their reproductive stage. All this material was housed at Natural Sciences Museum "Dr. Ángel Gallardo" of Rosario city, where it was processed, determined and stored.

Results: The examined flora comprises 128 species grouped in 109 genera and 42 families, of which 96 are native and three are considered endemic of Argentina. The families best represented considering species richness were Poaceae, Asteraceae and Fabaceae.

Conclusions: This floristic inventory allows to visualize the relevance of the study area as a suitable habitat for native and characteristic species that are absent in the surrounding croplands.

\section{KEY WORDS}

Floristic inventory, Pueblo Andino, Natural Water Reserve "Río Carcarañá", Santa Fe.

\section{RESUMEN}

Introducción y objetivos: La Reserva Hídrica Natural "Río Carcarañá", incluída en la Provincia Fitogeográfica Pampeana, constituye una de las escasas áreas protegidas del sur de la provincia de Santa Fe (Argentina), región profundamente modificada debido a la explotación agrícola y ganadera. Los estudios florísticos previos dentro de la reserva se refieren, fundamentalmente, a la zona de "El Espinillo" y la describen como un relicto de formaciones vegetales autóctonas con elementos florísticos de la Provincia Fitogeográfica del Espinal. El presente artículo tiene como objetivos realizar un relevamiento florístico dentro de los límites de la comuna de Pueblo Andino (departamento Iriondo) y estimar la distribución de especies a nivel de familias, identificar especies nativas y exóticas, como así también, reconocer las especies relevantes en función del contexto fitogeográfico en el que se circunscribe el área de estudio.

M\&M: Se realizaron nueve visitas y trabajo de campo al área entre 2014 y 2021 donde se colectaron ejemplares en estadio reproductivo, los cuales fueron procesados, determinados y luego depositados en el Museo de Ciencias Naturales "Dr. Ángel Gallardo" de la ciudad de Rosario.

Resultados: La flora examinada contiene 128 especies, agrupadas en 109 géneros y 42 familias, de las cuales 96 son nativas, y tres de ellas endémicas de Argentina. Las familias mejor representadas en cuanto a riqueza fueron Poaceae, Asteraceae y Fabaceae.

Conclusiones: El inventario florístico resultante permite visualizar la relevancia del área de estudio como sitio de albergue de especies vegetales nativas y características de la región, ausentes en las áreas cultivadas circundantes.

\section{Palabras clave}

Inventario florístico, Pueblo Andino, Reserva Hídrica Natural "Río Carcarañá", Santa Fe. 


\section{INTRODUCCIÓN}

La Reserva Hídrica Natural "Río Carcarañá” está localizada en el centro-sur de la provincia de Santa Fe (Argentina), en el noreste del departamento Iriondo, comprende una superficie de aproximadamente 10.020 hectáreas y constituye una de las escasas áreas protegidas del sur santafesino (Decreto provincial de creación $\mathrm{N}^{\circ} 1579$ de fecha 31 de mayo de 2012). Esta región se encuentra profundamente modificada como consecuencia de una larga historia de perturbaciones antrópicas que se remonta a más de un siglo; sometida primero a la ganadería y, posteriormente, a la explotación agrícola, sus ambientes naturales se vieron crecientemente degradados (Boccanelli, 2011; Menéndez \& Hilbert, 2013). En las últimas dos décadas se intensificó el uso y la explotación de sus suelos a partir de la aplicación de nuevos paquetes tecnológicos (siembra directa, organismos genéticamente modificados y uso intensivo de agroquímicos a gran escala) llevando a la destrucción casi completa de la vegetación autóctona de la región (Ghersa et al., 1998).

De acuerdo a Cabrera (1976) y Oyarzabal et al. (2018), desde el punto de vista fitogeográfico, el área de estudio se encuentra incluida en la Provincia Pampeana. Según Cabrera (1976) esta provincia se caracteriza por presentar como vegetación dominante praderas o pseudoestepas de gramíneas que forman densas matas entre las cuales crecen numerosas especies herbáceas de otras familias botánicas, acompañadas de algunos sufrútices y arbustos. Las comunidades vegetales más importantes de las pseudoestepas graminosas son los flechillares que se caracterizan por la presencia y abundancia de Nassella nessiana (Trin. \& Rupr.) Barkworth, Jarava plumosa (Spreng.) S.W.L. Jacobs \& J. Everett y Bothriochloa lagurioides (DC.) Herte, coincidiendo con las descripciones realizadas específicamente para la provincia de Santa Fe por Lewis (1981) y Pensiero et al. (2005). No obstante, cabe destacar que Cabrera (1976), al igual que Cabrera \& Willink (1980) y Pensiero et al. (2005), refiere a la presencia de bosques xerófilos con elementos florísticos del Espinal dentro de la Provincia Pampeana debido a condiciones ambientales particulares, como ocurre en ciertos sectores de las márgenes del río Carcarañá. Por su parte, Oyarzabal et al. (2018) proponen un mapa fisonómico-florístico de la vegetación espontánea de la Argentina que muestra la heterogeneidad dentro de provincias fitogeográficas. En este sentido, el área de estudio se encuentra incluida en la Unidad Pseudoestepa mesofítica de Bothriochloa lagurioides y Nassella spp., dentro de la cual los autores distinguen cuatro comunidades características; una comunidad zonal: pseudoestepa mesofítica; y tres comunidades azonales: pradera húmeda, estepa de halófitas y bosque xerofítico. Finalmente, cabe destacar que de acuerdo al esquema biogeográfico de la Argentina propuesto por Arana et al. (2021) el área de estudio queda incluída dentro de Provincia Pampeana, caracterizada por la presencia de sabanas con Poaceae intercaladas con bosque xérico; estos autores, a diferencia de Cabrera (1976) y Oyarzabal et al. (2018) refieren al Espinal como un distrito dentro de la Provincia Pampeana.

A pesar de que las comunidades de plantas nativas de esta región han sido reemplazadas paulatinamente por distintos cultivos (Boccanelli, 2011), espacios tales como los bordes de alambrados y las vías férreas, los costados de rutas y caminos, los márgenes de cursos de agua, entre otros, han quedado excluidos parcialmente de dicho proceso de agriculturización, conformando reservorios para la vegetación nativa $\mathrm{y}$, al mismo tiempo, una importante red de corredores biológicos (Biasatti et al., 2015). En el caso particular de las márgenes del río Carcaraná, la topografía, caracterizada por las ondulaciones del terreno, constituye uno de los factores limitantes más importantes para la realización de actividades productivas. Esta limitante favorece, en algunos sectores, la conservación de la estructura de la formación típica de la sabana propia de estos corredores. En este sentido, entre los valores de conservación de la reserva se destacan, ser un área de supervivencia de especies vegetales espontáneas completamente ausentes en el agroecosistema circundante, refugio de fauna y un corredor biológico de escala regional (Biasatti et al., 2017).

Los antecedentes de estudios florísticos dentro de la reserva se refieren, fundamentalmente, a la zona del "El Espinillo" ubicada en las proximidades de la ciudad de Casilda en el departamento Caseros. Dichos trabajos describen el área como un relicto de formaciones vegetales autóctonas, con presencia de elementos florísticos del Espinal (Biasatti et al., 1996, 2015, 2017; Benaglia et al., 1999). Si bien estos estudios son de importancia, aún se carece de un listado exhaustivo de las especies nativas y exóticas presentes en la reserva $\mathrm{y}$, cabe destacar, carecen de ejemplares depositados en herbarios de referencia. 


\section{E. Pedrero et al. - Inventario florístico de la Reserva Hídrica Natural "Río Carcarañá"}

El objetivo de este artículo es realizar un relevamiento florístico en la Comuna de Pueblo Andino, estimar la abundancia de especies por familia botánica, identificar las especies nativas y exóticas, y aquellas especies relevantes desde el punto de vista fitogeográfico.

\section{MATERIALES Y MÉtodos}

\section{Área de estudio}

La Reserva Hídrica Natural "Río Carcarañá", departamento Iriondo, provincia de Santa Fe (Argentina), comprende $300 \mathrm{~m}$ a ambas márgenes del río Carcarañá a lo largo de todo su trayecto dentro del territorio santafesino (aproximadamente $167 \mathrm{~km}$ ), alcanzando una superficie total de 10.020 hectáreas (Decreto $\mathrm{N}^{\circ} 1579 / 12$ ). Las localidades santafesinas que se asientan a las orillas del río Carcarañá comprenden a San José de la Esquina, Carcarañá, Lucio V. López, Pueblo Andino y Oliveros. El área de estudio relevada se encuentra dentro de los límites de la comuna de Pueblo Andino y se extiende desde los 3240'19,3” S $60^{\circ} 53^{\prime} 09,3$ " O hasta los 3241'43,3" S 6059'06,1" $\mathrm{O}$, abarcando $300 \mathrm{~m}$ de la margen izquierda del río acompañando las sinuosidades del mismo (Fig. 1). A lo largo del trayecto se pudo reconocer un mosaico de zonas con distintas fisonomías entre las que se destacan: bosques dominados por especies invasoras como Ligustrum lucidum W.T. Aiton y Gleditsia triacanthos L.; bosque con presencia de árboles y arbustos nativos como Celtis tala Gillies ex. Planch. y Schinus longifolia (Lindl.) Speg.; forestaciones de Eucalyptus spp.; campos de cultivos (soja, sorgo, maíz); pastizales con algunos elementos leñosos; y zonas bajas que forman lagunas en consonancia con el régimen hidrológico a lo largo del año (Fig. 2A-C).

\section{Métodos}

Se llevaron adelante nueve campañas de relevamiento en el denominado "Sendero de Bicicletas" a orillas del río Carcarañá, siete comprendidas entre marzo de 2014 y septiembre de 2016, una en diciembre de 2017 y una en marzo de 2021. Durante las distintas campañas se recolectaron ejemplares que fueron herborizados e identificados

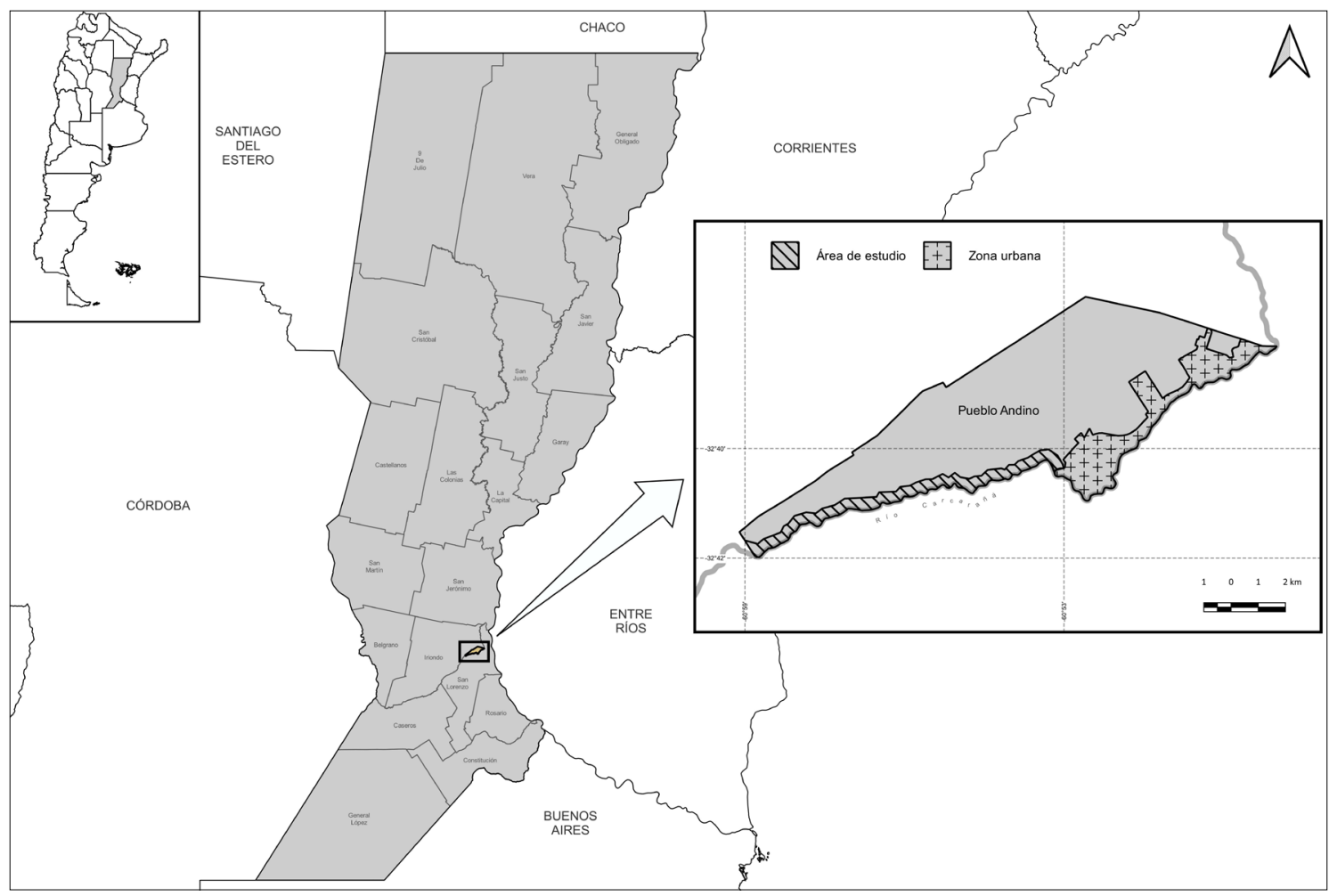

Fig. 1. Área de estudio en la Reserva Hídrica Natural "Río Carcarañá". 
Bol. Soc. Argent. Bot. 57 (1) 2022
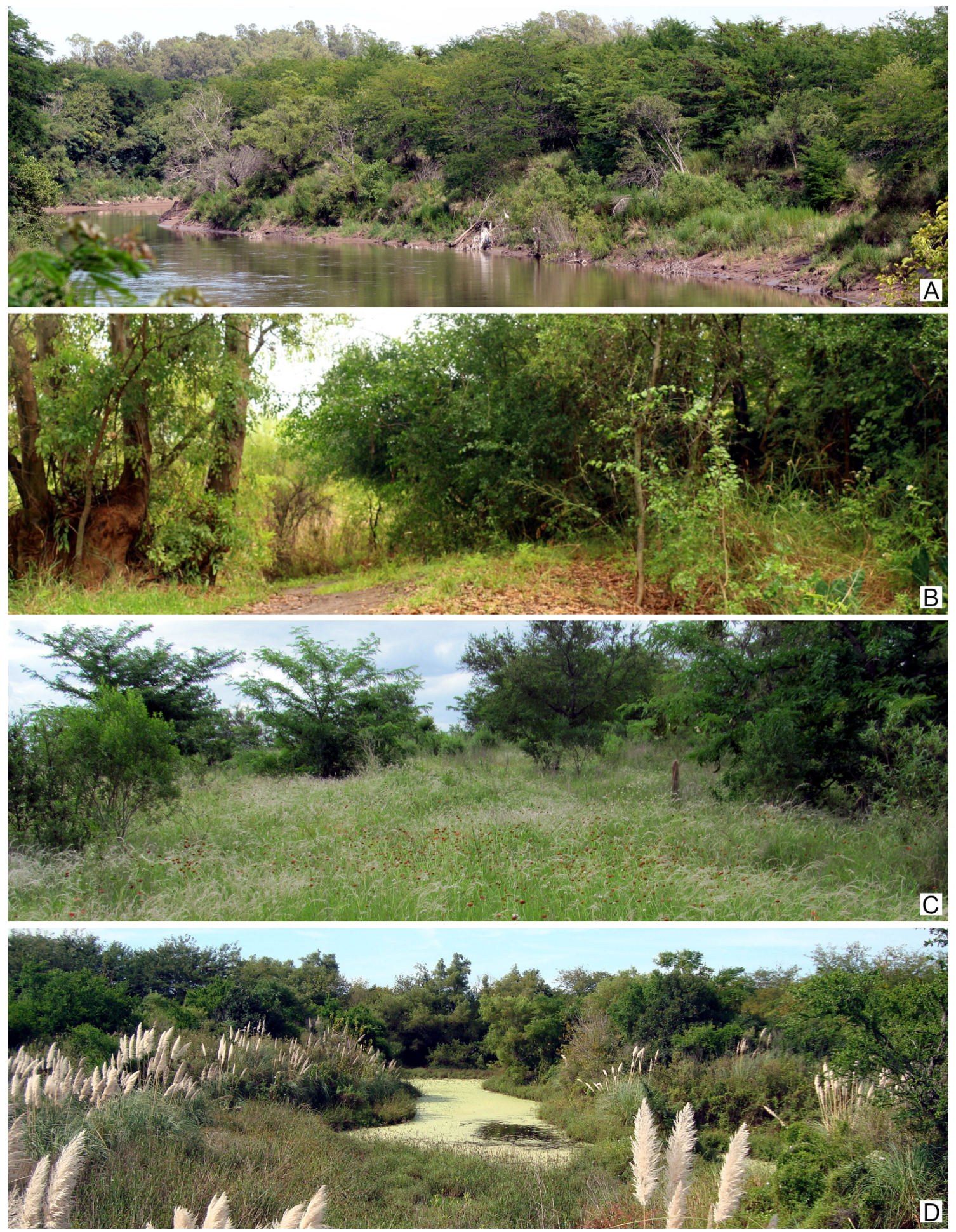

Fig. 2. Fisonomías representativas del área de estudio. A: Curso de agua y márgenes del río Carcarañá. B: Bosque. C: Pastizal con árboles dispersos. D: Zona baja con lagunas. Fotos de Germán Saigo y Violeta Di Doménica. 


\section{E. Pedrero et al. - Inventario florístico de la Reserva Hídrica Natural "Río Carcarañá"}

en gabinete. Las campañas se distribuyeron en distintas estaciones del año para obtener un muestrario de plantas que refleje la dinámica fenológica de las mismas. En relación a las últimas dos recorridas (2017 y 2021), su objetivo fue la recolección y observación de aquellas especies en las que se presentaron dudas en los relevamientos previos. Los ejemplares colectados fueron georreferenciados, fotografiados, rotulados y prensados en el campo. Las especies de la lista que no presentan número de inventario no fueron coleccionadas, aunque sí registradas fotográficamente e identificadas a campo; dichas fotografías fueron cargadas en la plataforma iNaturalist (https://www.inaturalist.org) donde alcanzaron grado de investigación, el respectivo DOI fue obtenido de la base de datos GBIF (https://www.gbif.org) y se indica en la tabla de resultados. Todo el material colectado fue depositado en el Museo de Ciencias Naturales "Dr. Ángel Gallardo" de la ciudad de Rosario, donde fue procesado.

Para la determinación taxonómica se utilizó la siguiente bibliografía: Flora de la provincia de Buenos Aires (Cabrera, 1965, 1970), Manual de la Flora de los Alrededores de Buenos Aires (Cabrera \& Zardini, 1978), Flora Ilustrada de Entre Ríos (Burkart, 1969, 1974, 1979; Burkart et al., 1987; Burkart \& Bacigalupo, 2005), Flora Vascular de la Provincia de Santa Fe
(Pensiero et al., 2005), Flora Argentina (Zuloaga et al., 2012a, 2012b; Zuloaga et al., 2014a, 2014b, 2015). Para la actualización de la nomenclatura científica se siguió la base de datos Flora del Cono Sur (IBODA, 2021).

Con la totalidad de las especies relevadas se confeccionó la lista florística correspondiente y se indicó en cada caso: la familia de pertenencia, el/los ejemplares de herbario de referencia, el estatus siguiendo los criterios de la base de datos Flora del Cono Sur (IBODA, 2021) y el hábito de crecimiento. Posteriormente, se estimó la distribución de especies en familias y se identificaron especies relevantes de acuerdo al contexto fitogeográfico en el que se circunscribe el área de estudio según Lewis \& Collantes (1973), Lewis (1981), Oyarzabal et al. (2018) y Arana et al. (2021).

\section{Resultados}

La lista florística resultante del relevamiento se presenta en la Tabla 1. El inventario consta de 128 especies de plantas vasculares reunidas en 109 géneros y 42 familias. Las familias mejor representadas en función del número de especies fueron: Poaceae (26 especies), Asteraceae (16 especies), Fabaceae (ocho especies), Solanaceae (ocho especies) y Verbenaceae (seis especies).

Tabla 1. Lista florística de la Reserva Hídrica Natural "Río Carcarañá" en la comuna de Pueblo Andino.

\begin{tabular}{|c|c|c|c|}
\hline Familias botánica y taxones & $\begin{array}{l}\text { Número de inventario de } \\
\text { ejemplares de herbario } \\
\text { de referencia / DOI }\end{array}$ & $\begin{array}{c}\text { Status de } \\
\text { distribución } \\
\text { para Argentina }\end{array}$ & Hábito \\
\hline \multicolumn{4}{|l|}{ Acanthaceae } \\
\hline Dicliptera squarrosa Nees & MG-B-V-00148/00195 & Nativa & Hierba \\
\hline \multicolumn{4}{|l|}{ Amaranthaceae } \\
\hline Alternanthera philoxeroides (Mart.) Griseb. & MG-B-V-00219/00258 & Nativa & Hierba \\
\hline Amaranthus hybridus L. & MG-B-V-00132 & Exótica & Hierba \\
\hline Iresine diffusa Humb. \& Bonpl. ex. Willd. & MG-B-V-00208 & Nativa & Subarbusto \\
\hline \multicolumn{4}{|l|}{ Amaryllidaceae } \\
\hline Zephyranthes filifolia Herb. ex Kraenzl. & MG-B-V-00238/00239 & Endémica & Hierba \\
\hline
\end{tabular}


Bol. Soc. Argent. Bot. 57 (1) 2022

\begin{tabular}{|c|c|c|c|}
\hline Familias botánica y taxones & $\begin{array}{l}\text { Número de inventario de } \\
\text { ejemplares de herbario } \\
\text { de referencia / DOI }\end{array}$ & $\begin{array}{c}\text { Status de } \\
\text { distribución } \\
\text { para Argentina }\end{array}$ & Hábito \\
\hline \multicolumn{4}{|l|}{ Anacardiaceae } \\
\hline Schinus longifolia (Lindl.) Speg var. longifolia & MG-B-V-00187/00188/00232 & Nativa & Árbol \\
\hline \multicolumn{4}{|l|}{ Apiaceae } \\
\hline Conium maculatum $\mathrm{L}$. & MG-B-V-00237/00253/00254 & Exótica & Hierba \\
\hline Eryngium horridum Malme & https://doi.org/10.15468/dl.htxewp & Nativa & Hierba \\
\hline Hydrocotyle bonariensis Lam. & MG-B-V-00216 & Nativa & Hierba \\
\hline \multicolumn{4}{|l|}{ Apocynaceae } \\
\hline Araujia brachystephana (Griseb.) Fontella \& Goyder & MG-B-V-00163/00241/00171 & Nativa & Enredadera \\
\hline Asclepias mellodora A. St.-Hil. & MG-B-V-00233 & Nativa & Hierba \\
\hline Oxypetalum solanoides Hook. \& Arn. & MG-B-V-00169 & Nativa & Hierba \\
\hline \multicolumn{4}{|l|}{ Asteraceae } \\
\hline Achyrocline flaccida (Weinm.) DC. & MG-B-V-00835 & Nativa & Hierba \\
\hline $\begin{array}{l}\text { Austroeupatorium inulifolium (Kunth) } \\
\text { R. M. King \& H. Rob. }\end{array}$ & MG-B-V-00126/00152 & Nativa & Arbusto \\
\hline Baccharis gilliesii A. Gray & MG-B-V-00190/00191 & Endémica & Subarbusto \\
\hline Baccharis notosergila Griseb. & MG-B-V-00827 & Nativa & Arbusto \\
\hline Baccharis punctulata DC. & MG-B-V-00125 & Nativa & Arbusto \\
\hline Baccharis salicifolia (Ruiz \& Pav.) Pers. & MG-B-V-00257 & Nativa & Arbusto \\
\hline Barrosoa cabrerae (B.L.Rob.) R.M.King \& H.Rob. & MG-B-V-00142/00143 & Endémica & Hierba \\
\hline Bidens pilosa L. var. pilosa & MG-B-V-00115/00197 & Nativa & Hierba \\
\hline Chromolaena ivifolia (L.) R.M. King \& H. Rob. & MG-B-V-00159 & Nativa & Subarbusto \\
\hline Cirsium vulgare (Savi) Ten. & MG-B-V-00207 & Naturalizada & Hierba \\
\hline Eclipta prostrata (L.) L. & MG-B-V-00131 & Nativa & Hierba \\
\hline $\begin{array}{l}\text { Hypochaeris albiflora (Kuntze) C. F. } \\
\text { Azêvedo-Gonçalves \& Matzenb. }\end{array}$ & MG-B-V-00116 & Nativa & Hierba \\
\hline $\begin{array}{l}\text { Gaillardia megapotamica (Spreng.) Baker } \\
\text { var. scabiosoides (Arn. ex DC.) Baker }\end{array}$ & MG-B-V-00129 & Nativa & Hierba \\
\hline Leucanthemum vulgare Lam. & MG-B-V-00153/00154/834 & Exótica & Hierba \\
\hline Microgyne trifurcata Less. & MG-B-V-00203/00212 & Nativa & Hierba \\
\hline Pascalia glauca Ortega & MG-B-V-00184 & Nativa & Hierba \\
\hline Senecio madagascariensis Poir. & MG-B-V-00176 & Exótica & Hierba \\
\hline Sonchus oleraceus L. & MG-B-V-00215 & Exótica & Hierba \\
\hline
\end{tabular}


E. Pedrero et al. - Inventario florístico de la Reserva Hídrica Natural "Río Carcarañá"

\begin{tabular}{|c|c|c|c|}
\hline Familias botánica y taxones & $\begin{array}{l}\text { Número de inventario de } \\
\text { ejemplares de herbario } \\
\text { de referencia / DOI }\end{array}$ & $\begin{array}{c}\text { Status de } \\
\text { distribución } \\
\text { para Argentina }\end{array}$ & Hábito \\
\hline Urolepis hecatantha (DC.) R. M. King \& H. Rob. & MG-B-V-00170 & Nativa & Hierba \\
\hline \multicolumn{4}{|l|}{ Brassicaceae } \\
\hline Brassica rapa L. & MG-B-V-00234/00235 & Exótica & Hierba \\
\hline Brassica nigra (L.) W.D.J. Koch & MG-B-V-00288/291 & Exótica & Hierba \\
\hline \multicolumn{4}{|l|}{ Bromeliaceae } \\
\hline Tillandsia aëranthos (Loisel.) L.B. Sm. & MG-B-V-00267 & Nativa & Hierba epífita \\
\hline Tillandsia recurvata (L.) L. & MG-B-V-00265/279 & Nativa & Hierba epífita \\
\hline Tillandsia tricholepis Baker & MG-B-V-00278 & Nativa & Hierba epífita \\
\hline \multicolumn{4}{|l|}{ Cactaceae } \\
\hline Opuntia elata Salm-Dyck var. obovata E. Walther & https://doi.org/10.15468/dl.htxewp & Nativa & Suculenta \\
\hline \multicolumn{4}{|l|}{ Calycearaceae } \\
\hline Acicarpha tribuloides Juss. & MG-B-V-00282 & Nativa & Hierba \\
\hline \multicolumn{4}{|l|}{ Campanulaceae } \\
\hline Wahlenbergia linarioides (Lam.) A. DC. & MG-B-V-00226 & Nativa & Hierba \\
\hline \multicolumn{4}{|l|}{ Cannabaceae } \\
\hline Celtis tala Gillies ex Planch. & MG-B-V-0082 a 00826 & Nativa & Árbol \\
\hline \multicolumn{4}{|l|}{ Caprifoliaceae } \\
\hline Dipsacus fullonum $\mathrm{L}$. & MG-B-V-00229 & Exótica & Hierba \\
\hline \multicolumn{4}{|l|}{ Caryophyllaceae } \\
\hline Silene antirrhina L. var. antirrhina & MG-B-V-00261/00264 & Exótica & Hierba \\
\hline \multicolumn{4}{|l|}{ Chenopodiaceae } \\
\hline Dysphania multifida (L.) Mosyakin \& Clemants & MG-B-V-00134 & Exótica & Hierba \\
\hline \multicolumn{4}{|l|}{ Commelinaceae } \\
\hline Commelina erecta L. & MG-B-V-00270 & Nativa & Hierba \\
\hline \multicolumn{4}{|l|}{ Convolvulaceae } \\
\hline Ipomoea cairica (L.) Sweet & MG-B-V-00137 & Nativa & Enredadera \\
\hline Ipomoea purpurea (L.) Roth & MG-B-V-00193/00198 & Nativa & Enredadera \\
\hline \multicolumn{4}{|l|}{ Cyperaceae } \\
\hline Cyperus aggregatus (Willd.) Endl. & MG-B-V-00124 & Nativa & Hierba \\
\hline Cyperus entrerianus Boeck. var. entrerianus & MG-B-V-00147 & Nativa & Hierba \\
\hline
\end{tabular}


Bol. Soc. Argent. Bot. 57 (1) 2022

\begin{tabular}{|c|c|c|c|}
\hline Familias botánica y taxones & $\begin{array}{l}\text { Número de inventario de } \\
\text { ejemplares de herbario } \\
\text { de referencia / DOI }\end{array}$ & $\begin{array}{l}\text { Status de } \\
\text { distribución } \\
\text { para Argentina }\end{array}$ & Hábito \\
\hline Cyperus rotundus $\mathrm{L}$. & MG-B-V-00118 & Nativa & Hierba \\
\hline \multicolumn{4}{|l|}{ Euphorbiaceae } \\
\hline Euphorbia dentata Michx. & MG-B-V-00135 & Nativa & Hierba \\
\hline \multicolumn{4}{|l|}{ Fabaceae } \\
\hline Gleditsia triacanthos $\mathrm{L}$. & MG-B-V-00144 & Exótica & Árbol \\
\hline $\begin{array}{l}\text { Geoffroea decorticans (Gillies ex. Hook. \& Arn.) } \\
\text { Burkart }\end{array}$ & https://doi.org/10.15468/dl.htxewp & Nativa & Árbol \\
\hline Medicago lupulina L. & MG-B-V-00255 & Exótica & Hierba \\
\hline Melilotus albus Desr. & MG-B-V-00113 & Exótica & Hierba \\
\hline Melilotus officinalis (L.) Lam. & MG-B-V-00114 & Exótica & Hierba \\
\hline Parkinsonia aculeata L. & MG-B-V-00174/00182/00252 & Nativa & Árbol \\
\hline Vachellia caven (Molina) Seigler \& Ebinger & MG-B-V-00864 & Nativa & Árbol \\
\hline Vigna luteola (Jacq.) Benth. & MG-B-V-00186/00839 & Nativa & Enredadera \\
\hline Trifolium repens $\mathrm{L}$. & MG-B-V-00185 & Exótica & Hierba \\
\hline \multicolumn{4}{|l|}{ Gentianaceae } \\
\hline Centaurium pulchellum (Sw.) Druse & MG-B-V-00160 & Exótica & Hierba \\
\hline \multicolumn{4}{|l|}{ Juncaceae } \\
\hline Juncus imbricatus Laharpe & MG-B-V-00218/00220/00224 & Nativa & Hierba \\
\hline \multicolumn{4}{|l|}{ Lamiaceae } \\
\hline Condea floribunda (Briq.) Harley \& J. F. B. Pastore & MG-B-V-00168 & Nativa & Hierba \\
\hline Leonurus japonicus Houtt. & MG-B-V-00263 & Exótica & Hierba \\
\hline \multicolumn{4}{|l|}{ Lythraceae } \\
\hline Cuphea glutinosa Cham. \& Schltdl. & MG-B-V-00274/00275 & Nativa & Hierba \\
\hline Heimia salicifolia Link & MG-B-V-00256 & Nativa & Subarbusto \\
\hline \multicolumn{4}{|l|}{ Malvaceae } \\
\hline Abutilon grandifolium (Willd.) Sweet & MG-B-V-00149/00150 & Nativa & Arbusto \\
\hline Pavonia sepium A. St.-Hill. & MG-B-V-00141 & Nativa & Arbusto \\
\hline Sida rhombifolia L. & MG-B-V-00117/00151 & Nativa & Subarbusto \\
\hline Sphaeralcea bonariensis (Cav.) Griseb. & MG-B-V-00138 & Nativa & Subarbusto \\
\hline Modiolastrum gilliesii (Steud.) Krapov. & MG-B-V-00242 & Nativa & Hierba \\
\hline Meliaceae & & & \\
\hline
\end{tabular}




\begin{tabular}{|c|c|c|c|}
\hline Familias botánica y taxones & $\begin{array}{l}\text { Número de inventario de } \\
\text { ejemplares de herbario } \\
\text { de referencia / DOI }\end{array}$ & $\begin{array}{c}\text { Status de } \\
\text { distribución } \\
\text { para Argentina }\end{array}$ & Hábito \\
\hline Melia azedarach L. & https://doi.org/10.15468/dl.htxewp & Exótica & Árbol \\
\hline \multicolumn{4}{|l|}{ Myrtaceae } \\
\hline Eucalyptus sp. & MG-B-V-00201 & Exótica & Árbol \\
\hline \multicolumn{4}{|l|}{ Oleaceae } \\
\hline Ligustrum lucidum W.T. Aiton & MG-B-V-00183 & Exótica & Árbol \\
\hline \multicolumn{4}{|l|}{ Onagraceae } \\
\hline Ludwigia bonariensis (Micheli) H. Hara & MG-B-V00831 & Nativa & Hierba \\
\hline Oenothera centaurifolia (Spach) Steud. & MG-B-V-00205/00236 & Nativa & Hierba \\
\hline Oenothera curtiflora W.L. Wagner \& Hoch & MG-B-V-00136 & Exótica & Hierba \\
\hline \multicolumn{4}{|l|}{ Oxalidaceae } \\
\hline Oxalis corniculata L. var. corniculata & MG-B-V-00283 & Nativa & Hierba \\
\hline Oxalis hispidula Zucc. & MG-B-V-00128 & Nativa & Hierba \\
\hline \multicolumn{4}{|l|}{ Passifloraceae } \\
\hline Passiflora caerulea L. & MG-B-V-00840 & Nativa & Enredadera \\
\hline \multicolumn{4}{|l|}{ Plantaginaceae } \\
\hline Plantago major L. & MG-B-V-00260 & Exótica & Hierba \\
\hline \multicolumn{4}{|l|}{ Poaceae } \\
\hline Aristida pallens Cav. & MG-B-V-00225 & Nativa & Hierba \\
\hline Arundo donax $\mathrm{L}$. & https://doi.org/10.15468/dl.htxewp & Exótica & Hierba \\
\hline Avena sativa L. var. sativa & MG-B-V-00243 & Exótica & Hierba \\
\hline Bothriochloa laguroides (DC.) Herter & MG-B-V-00121/00161 & Nativa & Hierba \\
\hline Bouteloua megapotamica (Spreng.) Kuntze & MG-B-V-00221/00222 & Nativa & Hierba \\
\hline Briza minor L. & MG-B-V-00245/00246 & Exótica & Hierba \\
\hline Bromus catharticus Vahl var. catharticus & MG-B-V-00189/00251 & Nativa & Hierba \\
\hline $\begin{array}{l}\text { Cortaderia selloana (Schult. \& Schult. f.) } \\
\text { Asch. \& Graebn. subsp. selloana }\end{array}$ & https://doi.org/10.15468/dl.htxewp & Nativa & Hierba \\
\hline Cynodon dactylon (L.) Pers. var. dactylon & MG-B-V-00829 & Exótica & Hierba \\
\hline $\begin{array}{l}\text { Cinnagrostis viridiflavescens (Poir.) P.M. Peterson, } \\
\text { Soreng, Romasch. \& Barberá var. viridiflavescens }\end{array}$ & MG-B-V-00162 & Nativa & Hierba \\
\hline Eleusine tristachya (Lam.) Lam. & MG-B-V00832 & Nativa & Hierba \\
\hline Eragrostis airoides Nees & MG-B-V-00196 & Nativa & Hierba \\
\hline Eustachys distichophylla (Lag.) Nees & MG-B-V-00837 & Nativa & Hierba \\
\hline
\end{tabular}


Bol. Soc. Argent. Bot. 57 (1) 2022

\begin{tabular}{|c|c|c|c|}
\hline Familias botánica y taxones & $\begin{array}{l}\text { Número de inventario de } \\
\text { ejemplares de herbario } \\
\text { de referencia / DOI }\end{array}$ & $\begin{array}{c}\text { Status de } \\
\text { distribución } \\
\text { para Argentina }\end{array}$ & Hábito \\
\hline Hordeum stenostachys Godr. & MG-B-V-00230 & Nativa & Hierba \\
\hline Lolium multiflorum Lam. & MG-B-V-00228 & Exótica & Hierba \\
\hline Nassella neesiana (Trin. \& Rupr.) Barkworth & MG-B-V-00248/00249 & Nativa & Hierba \\
\hline Panicum bergii Arechav. var. bergii & MG-B-V-00830 & Nativa & Hierba \\
\hline Panicum capillare L. & MG-B-V-00146 & Exótica & Hierba \\
\hline Paspalum dilatatum Poir. subsp. dilatatum & MG-B-V-00165/00210 & Nativa & Hierba \\
\hline Paspalum urvillei Steud. & MG-B-V-00192 & Nativa & Hierba \\
\hline Phalaris angusta Nees ex. Trin. & MG-B-V-00250 & Nativa & Hierba \\
\hline $\begin{array}{l}\text { Schizachyrium microstachyum (Desv. ex } \\
\text { Ham.) Roseng., B. R. Arrill. \& Izag. }\end{array}$ & MG-B-V-00202/00122 & Nativa & Hierba \\
\hline Setaria lachnea (Nees) Kunth & MG-B-V-00213 & Nativa & Hierba \\
\hline Setaria parviflora (Poir.) Kerguélen var. parviflora & MG-B-V-00120/00145/00209 & Nativa & Hierba \\
\hline Sorghum halepense L. (Pers.) var. halepense & MG-B-V-00166 & Exótica & Hierba \\
\hline Sporobolus indicus (L.) R. Br. var. indicus & MG-B-V-00119 & Nativa & Hierba \\
\hline \multicolumn{4}{|l|}{ Polygonaceae } \\
\hline Muehlenbeckia sagittifolia (Ortega) Meisn. & MG-B-V-00172/00179 & Nativa & Arbusto \\
\hline Rumex crispus L. & MG-B-V-00199/00240 & Exótica & Hierba \\
\hline \multicolumn{4}{|l|}{ Primulaceae } \\
\hline Lysimachia arvensis (L.) U. Manns \& Anderb. & MG-B-V-00247/269 & Exótica & Hierba \\
\hline \multicolumn{4}{|l|}{ Rosaceae } \\
\hline Duchesnea indica (Andrews) Focke & MG-B-V-00244 & Exótica & Hierba \\
\hline \multicolumn{4}{|l|}{ Salicaceae } \\
\hline Salix humboldtiana Willd. & MG-B-V-00828 & Nativa & Árbol \\
\hline \multicolumn{4}{|l|}{ Sapindaceae } \\
\hline Cardiospermum halicacabum L. var. halicacabum & MG-B-V-00838 & Nativa & Enredadera \\
\hline \multicolumn{4}{|l|}{ Solanaceae } \\
\hline Calibrachoa parviflora (Juss.) D’Arcy & MG-B-V-00352 & Nativa & Hierba \\
\hline Jaborosa integrifolia Lam. & MG-B-V-00271 & Nativa & Hierba \\
\hline Physalis pubescens L. & MG-B-V-00177 & Nativa & Hierba \\
\hline Physalis viscosa L. & MG-B-V-00231 & Nativa & Hierba \\
\hline Salpichroa origanifolia (Lam.) Baill. & MG-B-V-00167 & Nativa & Hierba \\
\hline
\end{tabular}




\section{E. Pedrero et al. - Inventario florístico de la Reserva Hídrica Natural "Río Carcarañá"}

\begin{tabular}{|c|c|c|c|}
\hline Familias botánica y taxones & $\begin{array}{l}\text { Número de inventario de } \\
\text { ejemplares de herbario } \\
\text { de referencia / DOI }\end{array}$ & $\begin{array}{c}\text { Status de } \\
\text { distribución } \\
\text { para Argentina }\end{array}$ & Hábito \\
\hline Solanum pseudocapsicum L. & MG-B-V-00266 & Nativa & Subarbusto \\
\hline Solanum chenopodioides Lam. & MG-B-V-00181 & Nativa & Hierba \\
\hline Solanum sisymbriifolium Lam. & MG-B-V-00194 & Nativa & Subarbusto \\
\hline \multicolumn{4}{|l|}{ Turneraceae } \\
\hline $\begin{array}{l}\text { Turnera sidoides L. subsp. pinnatifida (Juss. } \\
\text { ex Poir.) Arbo }\end{array}$ & MG-B-V-00276/00277 & Nativa & Hierba \\
\hline \multicolumn{4}{|l|}{ Verbenaceae } \\
\hline $\begin{array}{l}\text { Aloysia gratissima (Gillies \& Hook. ex Hook.) Tronc. } \\
\text { var. gratissima }\end{array}$ & MG-B-V-00175/00833 & Nativa & Arbusto \\
\hline Glandularia peruviana (L.) Small. & MG-B-V-00178/00180 & Nativa & Hierba \\
\hline Lantana megapotanica (Speng.) Tronc. & MG-B-V-00292 & Nativa & Arbusto \\
\hline $\begin{array}{l}\text { Phyla nodiflora (L.) Greene var. minor (Gillies \& } \\
\text { Hook. ex Hook.) N. O'Leary \& P. Peralta }\end{array}$ & MG-B-V-00227 & Nativa & Hierba \\
\hline $\begin{array}{l}\text { Phyla nodiflora (L.) Greene var. reptans (Kunth) } \\
\text { Moldenke }\end{array}$ & MG-B-V-00354/00355/00356 & Nativa & Hierba \\
\hline Pitraea cuneato-ovata (Cav.) Caro & MG-B-V-00206 & Nativa & Hierba \\
\hline Verbena litoralis Kunth var. litoralis & MG-B-V-00140 & Nativa & Hierba \\
\hline Verbena montevidensis Spreng. & MG-B-V-00127 & Nativa & Hierba \\
\hline
\end{tabular}

\section{Discusión}

En el inventario florístico aquí realizado se encuentran representados aproximadamente el $7 \%$ de las especies, el $15 \%$ de los géneros y el $28 \%$ de las familias registradas en la provincia de Santa Fe de acuerdo a Pensiero et al. (2005). Por otro lado, las cuatro familias más importantes en cuanto al número de especies (Poaceae, Asteraceae, Fabaceae y Solanaceae) son coincidentes con las registradas también por Pensiero et al. (2005) para la flora de Santa Fe.

Dentro del contexto en el que se sitúa el área relevada, cabe destacar que de las 128 especies registradas 96 son nativas $(75 \%)$, tres de las cuales son consideradas endémicas para la Argentina, según la base de datos Flora del Cono Sur (IBODA, 2021). Asimismo, es importante mencionar que las especies exóticas, Ligustrum lucidum y Gleditsia triacanthos, ya identificadas como un problema serio en otras comunidades riparias de la región (Biasatti et al. 2015), ocupan extensiones considerables dentro del área de estudio.

Respecto a la presencia de especies relevantes en función del contexto fitogeográfico, los componentes de la lista florística se contrastaron con las especies citadas para la unidades Pampa Santafesina y Espinal Periestépico, definidas para la provincia de Santa Fe por Lewis (1981) y Lewis \& Collantes (1973), la unidad Pseudoestepa Mesofítica de Bothriochloa lagurioides y Nassella spp., definida por Oyarzabal et al. (2018), y el distrito Pampeano Oriental definido por Arana et al. (2021).

Las especies nativas relevadas dentro del estrato arbóreo citadas por Lewis (1981) y Lewis \& Collantes (1973) para los bosques xerófilos del Espinal Periestépico son: Celtis tala, Vachellia caven (Molina) Seigler \& Ebinger, Geoffroea decorticans (Gillies ex. Hook. \& Arn.) Burkart, Schinus longifolia y Parkinsonia aculeata L.

Entre las especies nativas del estrato herbáceo descriptas para la Pampa Santafesina (Lewis, 1981) 
se encontraron: Nasella neesiana, Bothriochloa laguroides, Sporobolus indicus (L.) R. Br. var. indicus, Phyla nodiflora (L.) Greene var. minor (Gillies \& Hook. ex Hook.) N. O’Leary \& P. Peralta, Paspalum dilatatum Poir., Panicum bergii Arechav., Cinnagrostis viridiflavescens (Poir.) P.M. Peterson, Soreng, Romasch. \& Barberá var. viridiflavescens, Setaria parviflora (Poir.) Kerguélen var. parviflora, Hordeum stenostachys Godr., Acicarpha tribuloides Juss. y Modiolastrum gilliesii (Steud.) Krapov. Cabe destacar que las cuatro primeras especies mencionadas fueron utilizadas por Lewis (1981) para caracterizar a los distintos espartillares dentro de la unidad Pampa Santafesina. Asimismo, N. neesiana y $B$. laguroides son especies constituyentes de la comunidad clímax Pseudoestepas de Flechillas referida por Cabrera (1976) para el distrito Pampeano Oriental.

Por otra parte, se detallan a continuación las especies presentes en el inventario correspondientes a la vegetación espontánea característica de la unidad Pseudoestepa Mesofítica de Bothriochloa lagurioides y Nassella spp. definida por Oyarzabal et al. (2018), señalando además, la comunidad de pertenencia definida por dichos autores. Dentro de la comunidad zonal Pseudoestepa Mesofítica se identificaron: Bothriochloa laguroides (señalada por los autores como una de las especies dominantes) y Nassella neesiana, además los autores hacen mención de forma general a la presencia de distintas especies de Baccharis L. y Verbena L. En este sentido, en el inventario se encontraron: B. gilliesii A. Gray, B. punctulata DC., B. salicifolia (Ruiz \& Pav.) Pers. y $B$. notosergila Griseb.; V. litoralis Kunth var. litoralis y $V$. montevidensis Spreng., respectivamente; dentro de la comunidad azonal Pradera Húmeda se reconocieron: Paspalum dilatatum, Setaria parviflora, Sporobolus indicus (L.) R. Br.. Mientras que dentro de los géneros Cyperus L. y Juncus L. (los autores no hacen referencia a especies) se encontraron: C. aggregatus (Willd.) Endl., C. entrerianus Boeck., C. rotundus L. y J. imbricatus Laharpe, respectivamente; y finalmente, dentro de la comunidad azonal Bosque Xerofítico de Celtis ehrenbergian" se reconoció Celtis tala.

Por último, las especies presentes en el inventario correspondientes al distrito Pampeano Oriental definido por Arana et. al (2021) son las siguientes: Nassella neesiana, Bothriochloa laguroides, Heimia salicifolia (Kunth) Link, Celtis tala y Schinus longifolia.

\section{Conclusiones}

El análisis de los componentes del inventario permite visualizar que, si bien el área de estudio se encuentra sumamente modificada, desde el punto de vista fitogeográfico aún conserva elementos florísticos característicos de la zona: se registra la presencia de numerosas especies propias de la Pampa y elementos leñosos característicos del Espinal. Los resultados muestran la relevancia del área de estudio como sitio de albergue de especies nativas y representativas de la región que están ausentes en las áreas cultivadas circundantes.

Además, cabe destacar la importancia de los inventarios florísticos para conocer y analizar distintos aspectos asociados a la diversidad vegetal. En este sentido, el presente inventario cobra mayor relevancia dado que el área de estudio se caracteriza por la pérdida de la mayoría de sus ambientes naturales representativos, además de formar parte de un área natural protegida. De esta manera, el artículo contribuye a ampliar y actualizar los conocimientos acerca de la flora del sur de la provincia de Santa Fe y, en particular, de la Reserva Hídrica Natural "Río Carcarañá". En este contexto, dicho inventario podría configurar una herramienta fundamental para la elaboración de planes de manejo y monitoreo imprescindibles para llevar adelante estrategias de conservación en dicha área natural protegida.

\section{Contribución DE LOS AUTORES}

Todos los autores han realizado conjuntamente y a partes iguales la colecta de datos, su interpretación y la redacción del manuscrito.

\section{Agradecimientos}

A María Belén Sender y Verónica Carolina Albute por haber impulsado los trabajos de relevamientos biológicos en el área de estudio y por su participación en las primeras campañas de 


\section{E. Pedrero et al. - Inventario florístico de la Reserva Hídrica Natural "Río Carcarañá"}

colecta. A Leonardo Tempesta por su compromiso con el proyecto y por poner a disposición instalaciones y recursos para las primeras salidas de campo. A Mariela Antola, Mauricio Taborda, Bárbara Delgado, Lucía Levy Fachena, Claudia Duque, Eugenio Álvarez Arnesi, Franco Cirione, Violeta Di Doménica, Lía Martin, Paula San Pedro, Sofía Auil, Maia Kaminsky y Natalia Cavalieri, por su cooperación en las campañas de colecta y en el trabajo de gabinete. A Darién Prado por sus comentarios. A Hernán Maturo por sus aportes y orientar el trabajo de campo. A Débora Chamorro por colaborar con la identificación de los Celtis. Un agradecimiento especial a Luis Oakley y Virginia Mogni, quienes colaboraron con las determinaciones taxonómicas y estuvieron siempre predispuestos para responder nuestras consultas. $\mathrm{Y}$ a Eugenia Montani, por las sugerencias que ayudaron a enriquecer el manuscrito.

\section{Biblografía}

ARANA, M. D., E. NATALE, N. FERRETTI, G. ROMANO, A. OGGERO, G. MARTÍNEZ, P. POSADAS \& J. J. MORRONE. 2021. Esquema biogeográfico de la República Argentina. Tucumán: Fundación Miguel Lillo. Argentina.

BENAGLIA, A., N. R. BIASATTI \& M. C. ROMANO. 1999. Las variaciones del ambiente y su influencia sobre la biodiversidad en "El Espinillo" - Santa $\mathrm{Fe}$, Argentina -. Relaciones entre la avifauna y la vegetación. UNR Ambiental 3: 63-78.

BIASATTI, N. R., L. MARC, P. RIMOLDI \& E. SPIAGGI. 2017. Los valores ambientales de ecosistemas relictuales en la pampa húmeda: caso de "El Espinillo" y el tramo medio del río Carcarañá en Santa Fe. Rosario: UNR Editora. Argentina.

BIASATTI, N. R., J. C. ROZZATTI, B. FANDIÑO, A. PAUTASSO, E. MOSSO, G. MARTELEUR, N. ALGARAÑAZ, A. GIRAUDO, C. CHIARULLI, M. ROMANO, P. RAMÍREZ LLORENS \& L. VALLEJOS. 2015. Las ecorregiones, su conservación y las áreas naturales protegidas de la provincia de Santa Fe. 1. ${ }^{a}$ ed. Santa Fe: Imprenta UNL. Argentina.

BIASATTI, N. R., M. C. ROMANO, E. F. PIRE \& J. FERNÁNDEZ. 1996. Desarrollo teórico - práctico para la implementación de áreas protegidas en el sur de la provincia de Santa Fe, Argentina. Estudio de prefactibilidad del proyecto de área protegida en la zona de "El Espinillo". Depto. Caseros, Santa Fe, Argentina. UNR Ambiental 2: 92-102.

BOCCANELLI, S. I. 2011. Dinámica de la vegetación luego del abandono de campos agrícolas en el sur de la Provincia de Santa Fe, Argentina. Phyton 80: 227-29.

BURKART, A. E. 1969. Flora ilustrada de Entre Ríos (Argentina). Parte 2: Gramineas: La familia botánica de los pastos. Buenos Aires: Instituto Nacional de Tecnología Agropecuaria. Argentina.

BURKART, A. E. 1974. Flora ilustrada de Entre Ríos (Argentina). Parte 6: Dicotiledóneas Metaclamídeas (Gamopétalas). B: Rubiales, Cucurbitales, Campanulales (incluso Compuestas). Buenos Aires: Instituto Nacional de Tecnología Agropecuaria.

BURKART, A. E. 1979. Flora ilustrada de Entre Ríos (Argentina). Parte 5: Dicotiledóneas Metaclamídeas (Gamopétalas). A: Primulales a Plantaginales. Buenos Aires: Instituto Nacional de Tecnología Agropecuaria.

BURKART, A. E. \& N. M. BACIGALUPO. 2005. Flora ilustrada de Entre Ríos (Argentina). Parte 4: Dicotiledóneas Arquiclamídeas. B: Geraniales a Umbelliflorales. Buenos Aires: Instituto Nacional de Tecnología Agropecuaria.

BURKART, A. E., N. S. TRONCOSO DE BURKART \& N. M. BACIGALUPO. 1987. Flora ilustrada de Entre Ríos (Argentina). Parte 3: Dicotiledóneas Metaclamídeas. A: Salicales a Rosales (incluso Leguminosas). Buenos Aires: Instituto Nacional de Tecnología Agropecuaria.

CABRERA, A. L. 1965. Flora de la provincia de Buenos Aires. Tomo 4 parte 5. Buenos Aires: Instituto Nacional de Tecnología Agropecuaria.

CABRERA, A. L. 1970. Flora de la provincia de Buenos Aires. Tomo 4 parte 2. Buenos Aires: Instituto Nacional de Tecnología Agropecuaria.

CABRERA, A. L. 1976. Enciclopedia argentina de agricultura y jardinería. Fasciculo 1: Regiones fitogeográficas argentinas. Buenos Aires: Acme.

CABRERA, A. L. \& A. WILLINK. 1980. Biogeografia de América Latina. 2. ${ }^{\mathrm{a}}$ ed. Serie de Biología. Monografía $\mathrm{n}^{\circ}$ 13. Washington DC: Secretaría General de la Organización de los Estados Americanos, Programa Regional de Desarrollo Científico y Tecnológico.

CABRERA A. L. \& E. M. ZARDINI. 1978. Manual de la flora de los alrededores de Buenos Aires. 2. ${ }^{a}$ ed. Buenos Aires: Editorial Acme S.A.C.I. 
GBIF.org. GBIF Occurrence [online]. Disponible en: https://doi.org/10.15468/dl.htxewp [Acceso: 23 Noviembre 2021].

GHERSA, C. M., M. A. MARTÍNEZ-GHERSA \& R. J. C. LEÓN. 1998. Cambios en el paisaje pampeano. $\mathrm{Su}$ efecto sobre los sistemas de soporte de vida. En: O. T. SOLBRIG, L. VAINESMAN, J. ADELMAN, G. E. ALCHOURON, C. F. ARAGÓN, R. R. CASAS \& C. P. TIMMER (eds.). Hacia una agricultura más productiva y sostenible en la Pampa Argentina: una visión general prospectiva interdisciplinaria. Buenos Aires: Orientación Gráfica Editora.

IBODA. 2021. Flora del Cono Sur [online]. Disponible en http://www. darwin.edu.ar/Proyectos/ FloraArgentina/fa.htm [Acceso: 10 noviembre 2021].

iNaturalist. [online]. Available from https://www. inaturalist.org. [Acceso: 23 november 2021].

LEWIS, J. P. 1981. La vegetación de la provincia de Santa Fe. GAEA 9: 121-48.

LEWIS, J. P. \& M. B. COLLANTES. 1973. El espinal periestépico. Ciencia e Investigación 29: 360-77.

MENÉNDEZ, J. E. \& J. A. HILBERT. 2013. Cuantificación y uso de biomasa de residuos de cultivos en Argentina para bioenergía. Buenos Aires: Instituto Nacional de Tecnología Agropecuaria.

OYARZABAL, M., J. CLAVIJO, L. J. OAKLEY \& F. BIGANZOLI. 2018. Unidades de Vegetación de la Argentina. Ecología Austral 28: 40-63. https://doi.org/10.25260/EA.18.28.1.0.399.

PENSIERO, J., H. GUTIERREZ, A. LUCHETTI, E. EXNER, V. KERN, E. BRNICH, L. OAKLEY, D.
PRADO \& J. LEWIS. 2005. Flora vascular de la provincia de Santa Fe. Claves para el reconocimiento de las familias y géneros. Catálogo sistemático de las especies. 1. ${ }^{\text {a }}$ ed. Santa Fe: Universidad Nacional del Litoral.

ZULOAGA, F. O., M. J. BELGRANO \& A. M. R. ANTON. 2014a. Flora Argentina: flora vascular de la República Argentina 7(3): Dicotyledoneae, Asteraceae (Senecioneae a Vernonieae). 1. ${ }^{\mathrm{a}}$ ed. Vol. 7. San Isidro: Instituto de Botánica Darwinion. https://doi.org/10.2307/j.ctt16xwb7t.

ZULOAGA, F. O., M. J. BELGRANO \& A. M. R. ANTON. 2014b. Flora vascular de la República Argentina 7(1): Dicotyledoneae-Asteraceae (Anthemideae a Gnaphalieae). 1. ${ }^{\mathrm{a}}$ ed. Vol. 7. San Isidro: Instituto de Botánica Darwinion. https://doi.org/10.2307/j.ctt17mvkg1.

ZULOAGA, F. O., M. J. BELGRANO \& A. M. R. ANTON. 2015. Flora vascular de la República Argentina 7(2): Dicotyledoneae-Asteraceae (Cichorieae, Helenieae-Mutisieae). 1. ${ }^{\mathrm{a}}$ ed. Vol. 7. San Isidro: Instituto de Botánica Darwinion. https://doi.org/10.2307/j.ctt19dzdnt.

ZULOAGA, F. O., Z. E. RÚGOLO \& A. M. R. ANTON. 2012a. Flora vascular de la República Argentina 3(1): Monocotyledoneae, Poaceae (Aristidoideae a Pharoideae). 1. a ed. Vol. 3. Córdoba: Gráficamente Ediciones.

ZULOAGA, F. O., Z. E. RÚGOLO \& A. M. R. ANTON. 2012b. Flora vascular de la República Argentina 3(2): Monocotyledoneae, Poaceae (Pooideae). 1. ed. Vol. 3. Córdoba: Gráficamente Ediciones. 